\title{
POLIFARMÁCIA: USO DE MEDICAMENTOS POR IDOSOS
}

\author{
POLYPHARMACY: USE OF MEDICINES FOR ELDERLY
}

${ }^{1}$ Leticia Martins Melo, ${ }^{2}$ Bruno Nunes do Vale.

RESUMO

Introdução: A melhoria da qualidade de vida populacional proporciona o crescimento na faixa etária senil em todo mundo. Esse fato deve-se a diminuição dos índices de mortalidade, natalidade, além do controle de enfermidades crônicas e degenerativas. Desse modo, os idosos utilizam diariamente cerca de dois ou mais medicamentos, caracterizando polifarmácia. Objetivo: Verificar por meio de literatura os principais efeitos colaterais e reações adversas gerados em idosos a partir da utilização da polifarmácia, além das principais doenças acometidas por essa faixa etária. Material e Métodos: Pesquisa de caráter bibliográfico, de natureza descritiva, com abordagem qualitativa. Resultados e Discussão: Atualmente, no Brasil, considera-se idosa pessoas a partir dos 60 anos de idade. O amplo uso de medicamentos pode gerar consequências que coloquem em risco a segurança do paciente, entretanto, a terapia medicamentosa ainda é uma das escolhas para cuidado com a pessoa idosa. A atenção farmacêutica é extremamente importante no processo de utilização apropriada de fármacos para os idosos, pois proporciona um acompanhamento farmacoterapêutico adequado. Conclusão: A exposição a múltiplos fármacos, praticada por pacientes idosos tem se revelado principal fator arrolado a segurança da terapia medicamentosa, no tocante a ocorrência de reações adversas e interações. $O$ envelhecimento acarreta no aparecimento de diversas doenças como diabetes, hipertensão, osteoporose, que necessitam de tratamento contínuo, levando o idoso ao uso de vários medicamentos concomitantemente. No intuito de alcançar o sucesso terapêutico, o farmacêutico atua na atenção farmacêutica ao paciente senil, orientando quanto a sua terapia a ser seguida.

Descritores: Polifarmácia. Idosos. Atenção Farmacêutica. Reações adversas a medicamentos.

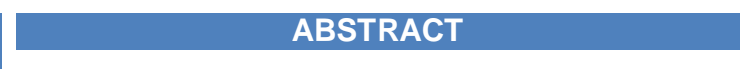

Introduction: Improving the quality of population life provides the growth in the senile age range worldwide. This fact is due to the decrease in mortality, birth rates, and control of chronic and degenerative diseases. In this way, the elderly use a daily about two or more medicines, featuring polypharmacy. Objective: To check through literature the main side effects and adverse reactions generated in elderly people from the use of polypharmacy in addition to the main diseases involved by this age group. Methodology: Bibliographical character research, descriptive nature, with a qualitative approach. Development: Currently, in Brazil, it considers itself elderly people from the 60 years of age. The extensive use of medicines can generate consequences that endanger patient safety, however, drug therapy is still one of the choices for caring for the elderly person. Pharmaceutical attention is extremely important in the process of proper use of pharmaceuticals for the elderly, because it provides an appropriate pharmacotherapeutic accompaniment. Conclusion: The exposure to multiple drugs, practiced by elderly patients has revealed the main factor in the safety of drug therapy, as regards the occurrence of adverse reactions and interactions. Aging involves the emergence of several diseases such as diabetes, hypertension, osteoporosis, which require continuous treatment, leading the elderly to use several medicines simultaneously. In order to achieve therapeutic success, the pharmacist operates in pharmaceutical attention to the senile patient, guiding as to his therapy to be followed.

\section{Descriptors: Polypharmacy. Elderly.} Pharmaceutical attention. Adverse drug reactions.

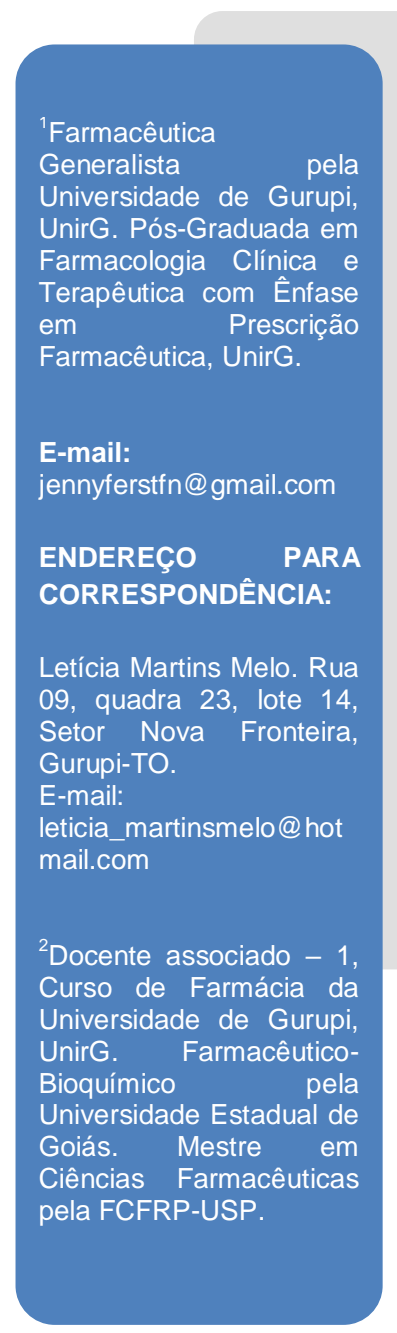

\section{INTRODUÇÃO}

A mídia juntamente com o marketing de medicamentos, aliados ao crescimento da indústria farmacêutica contribuem de forma direta e indireta para a elevação do número de prescrições de tratamentos farmacoterapêuticos por profissionais de saúde, propiciando o uso de múltiplos fármacos por idosos ${ }^{26}$.
A polifarmácia, uso por qualquer paciente de cinco ou mais medicamentos, incluindo-se aqueles oriundos de automedicação. Essa prática tem sido relacionada aos idosos, pois, o controle de doenças crônicas como diabetes, hipertensão, hipercolesterolemia, exige a tomada de diversos fármacos ${ }^{1,5,19}$.

Com o avançar da idade, acarretando no aumento e prevalência de doenças crônicas, verifica-se 0 aumento do consumo de 
Recebido: 25 junho 2017. Aceito: 14 dezembro 2018. Revista Amazônia Science \& Health - 2018 Out/Dez.
Melo, LM. Do Vale, BN.

POLIFARMÁCIA: USO DE MEDICAMENTOS POR IDOSOS medicamentos pela população idosa. Geralmente, os pacientes senis apresentam mais de uma patologia, o que contribui para polifarmácia, o que pode ocasionar reações e efeitos adversos ${ }^{1,2}$

O envelhecimento da população é uma realidade em todo o mundo. No Brasil, a amostra da população da terceira idade era 14,1 milhões em 2002, estima-se que este montante chegará ao número de 33,4 milhões em 2025. Considera-se grande avanço, considerando um país em desenvolvimento, entretanto, manter a qualidade de vida acarretará um grande desafio ${ }^{2,3,4}$. Ainda nesse contexto, estima-se que em 2050 mais de dois bilhões de pessoas terão atingido a melhor idade, o que pressupõe aumento no número de medicamentos utilizados para manutenção da saúde ${ }^{3,5}$.

O uso de medicamentos além do que está clinicamente indicado, pode gerar consequências que coloquem em risco a segurança do paciente, entretanto, a terapia farmacológica ainda é uma das melhores alternativas para cuidado com a saúde de uma pessoa idosa $\mathrm{i}^{2,3,9}$.

O uso irracional de medicamentos e a automedicação estão intimamente ligados a polifarmácia, principalmente sobre aqueles medicamentos que exigem prescrição médica, medicamentos injetáveis e a intercambialidade de medicamentos $^{6,15}$.

Desse modo, tendo em vista que a população senil se constitui de saúde frágil e necessita atenção especial por parte dos profissionais de saúde, pensando nisto, este trabalho teve por objetivo verificar por meio de literatura os principais efeitos colaterais e reações adversas gerados em idosos a partir da utilização de diversos medicamentos concomitantemente, além das principais doenças acometidas por essa faixa etária.

\section{MATERIAL E METODOS}

Trata-se de uma pesquisa de caráter bibliográfico, de natureza descritiva, com abordagem qualitativa. $\mathrm{O}$ trajeto metodológico escolhido, foi de natureza exploratória e descritiva, o que possibilitou alcançar o objetivo proposto.

O estudo ocorreu por meio de análise de estudos científicos buscados em revistas e sites indexados, como Scielo (Scientific Electronic Library Online), Lilacs (Literatura Latino-Americana e do Caribe de Informação em Ciências da Saúde), Bireme, Pubmed/Medline (Medical Literature Analysis and Retrieval System Online), Biblioteca Virtual da Saúde, num recorte temporal entre os anos de 2007 a 2017. As palavras-chave utilizadas para pesquisa foram: "Polifarmácia", "Atenção Farmacêutica", "Uso de Medicamentos por Idosos".

Foram incluídos todos os artigos científicos, dissertações, teses e resoluções que abordavam a polifarmácia praticada por idosos, no recorte temporal de 2007 a 2017 e que incluíam todos os aspectos referentes ao tema e suas complicações. Desses, foram encontrados 2.970 artigos, sendo excluídos todos os artigos científicos que não abordavam o tema, ou anteriores ao ano de 2007 , ou que não incluam os objetivos a serem pesquisados, totalizando 26 artigos selecionados para compor esta pesquisa.

\section{RESUTADOS E DISCUSSĀO}

O processo de envelhecimento é determinado pela elevação da idade do indivíduo, caracterizando uma fase com mudanças comportamentais, fisiológicas e sociais. Atualmente, no Brasil, considera-se idosa a pessoa que está acima dos 60 anos de idade ${ }^{1,19}$. O envelhecimento da população brasileira tem sido bastante discutido por diversos autores, uma vez que os idosos dependem cada vez mais dos serviços públicos de saúde, gerando maior gasto. A terceira idade requer maior atenção, tendo em vista a vulnerabilidade de sua condição de saúde, necessitando auxílio especializado e baseado em suas particularidades psicofisiológicas $^{9,12}$.

As alterações fisiológicas e patologias fazem com que os idosos necessitem de variados medicamentos, favorecendo com isso o uso da polifarmácia e automedicação. A polifarmacoterapia pode ser considerada como a quantidade de diversos fármacos ingeridos por um mesmo indivíduo, neste sentido pode ser categorizada como pequena, quando de dois a três fármacos; moderada, quando de quatro a cinco; e grande, quando acima de cinco fármacos ${ }^{6,7,24}$.

Assim, a exposição a múltiplos fármacos pode contribuir para 0 uso de medicamentos inadequados e não essenciais para o tratamento. Cria também uma barreira para a adesão ao tratamento, na medida em que torna mais complexos os esquemas terapêuticos, favorecendo com isso as interações medicamentosas e reações adversas $^{8,21}$.

De acordo com a Organização Mundial de Saúde, amplo espectro de medicamentos são prescritos ou dispensados de forma inadequada, e ainda, um número elevado de pacientes consomem seus medicamentos de maneira incorreta ou desistem do tratamento precocemente ${ }^{11,18}$.

O termo polifarmácia é definido como o uso de medicamentos além dos clinicamente indicados ou o uso de cinco ou mais fármacos ao mesmo tempo ou ainda, a exposição a múltiplos fármacos. Essa tendência aumenta a cada dia, tornando-se uma questão bastante rotineira. Além disso, essa definição tem obtido outras conotações tais como uso de medicamentos indevidos, evento de interação medicamentosa e efeito adverso de uma droga tratada com outra droga ${ }^{10,13}$. 
Recebido: 25 junho 2017. Aceito: 14 dezembro 2018. Revista Amazônia Science \& Health - 2018 Out/Dez.
Melo, LM. Do Vale, BN.

POLIFARMÁCIA: USO DE MEDICAMENTOS POR IDOSOS
As reações adversas a medicamentos em idosos é sabidamente um importante problema de saúde pública, cuja relação de risco é bem estabelecida. Assim, ao utilizar diversos fármacos simultâneos é possível que o idoso sinta reações indesejadas e consequentemente procure um serviço de saúde, ocasionando uma internação hospitalar. Na maioria das vezes, esse sintoma é tratado como uma nova patologia e não como uma reação adversa, fazendo com que o idoso volte para casa com outros medicamentos, o que no futuro pode ocasionar em vários desfechos clínicos ${ }^{1,22}$.

O exercício da polifarmácia é considerado multifatorial, sofrendo influência, sobretudo pelas doenças crônicas e por patologias consequentes do envelhecimento, sendo provável que esses fatores motivem o uso de vários medicamentos, incluindo também a automedicação $0^{7,11,24}$.

De acordo com Medeiros-Souza e colaboradores $^{10}$, a terapia multimedicamentosa é um respeitável apontador da condição da atenção à saúde dos idosos independente dos parâmetros que serão avaliados na sua definição. O essencial é que se procure o diagnóstico de suas causas e se defina os possíveis riscos para o idoso, de modo que melhore a condição da terapêutica medicamentosa desse grupo tão frágil.

Com o envelhecimento, é comum a manifestação de doenças crônicas, como osteoporose, incontinência urinária, diminuição da acuidade visual e auditiva, depressão, diabetes, acidente vascular cerebral, neoplasias, hipertensão arterial, entre outras doenças ${ }^{3,9,22}$. Necessitando de maior comprometimento por parte das equipes de saúde, além de ações comportamentais saudáveis que incluam o idoso e sua família. No decorrer desse processo, aumenta consideravelmente a busca por melhores condições de saúde, tornando clara a necessidade de verificar a prevalência dessas morbidades ${ }^{12,25}$.

Bueno e colaboradores ${ }^{7}$ mostram em sua pesquisa perfil de uso de medicamentos por idosos assistidos pelo Programa de Atenção ao Idoso da UNIJUÍ, no estado do Rio Grande do Sul, que as doenças que mais acometiam a população pesquisada eram as doenças do sistema nervoso, cardiovascular, digestores e do músculo esquelético.

A presença de diversas doenças pode ser dependente da qualidade e do acesso aos serviços de saúde e dos diagnósticos clínicos. Assim é importante que esse paciente tenha contato com o profissional de saúde para estar ciente de sua condição e, assim ser submetido a um tratamento medicamentoso adequado ${ }^{14,20,23}$.

A atenção farmacêutica (AF) é entendida como uma ação do profissional farmacêutico, onde o principal beneficiário é o paciente, diminuindo o risco de reações adversas e interações medicamentosas, obtendo-se o máximo de benefícios farmacoterapêuticos possíveis, além do uso racional de medicamentos ${ }^{1,15}$.

A atuação farmacêutica onde o profissional assume em primeiro lugar o bem-estar do paciente, auxiliando o prescritor a escolher adequadamente 0 medicamento, contribui diretamente para uma adesão maior ao tratamento e para a abrangência dos efeitos desejados. Muitos medicamentos, ainda que sejam apropriados, necessitam de um acompanhamento farmacoterapêutico ${ }^{7,12,14}$.

A inserção de um programa de um plano de assistência farmacêutica ao paciente, que relaciona a quantidade de medicamento fornecido a administração do mesmo, ajuda na identificação e relação a adesão a terapia prescrita pelo médico ${ }^{16,23}$.

A AF é extremamente importante no processo de utilização adequada de medicamentos para os idosos, pois esta propicia um acompanhamento farmacoterapêutico baseado na melhoria da qualidade de vida do paciente, diminuindo a utilização de fármacos sem prescrição médica, evitando reações e efeitos indesejados decorrentes de terapias equivocadas ${ }^{7,11,20}$.

O Brasil apresenta atualmente um momento de reestruturação na área dos medicamentos, podendo esse cenário ser visualizado tanto no setor público como privado. Através da AF que o profissional farmacêutico assume a responsabilidade de cuidar do paciente, esclarecendo as dúvidas do mesmo, mantendo uma relação de confiança mútua ${ }^{17,18}$.

\section{CONCLUSÁO}

Por meio do levantamento bibliográfico, foi possível verificar que a senilidade é uma condição cada vez mais presente em todo o mundo, fato que comprova a melhoria da qualidade de vida dos indivíduos. Assim, é necessário o uso de mais terapias, inclusive medicamentosa, para que se tenha bem-estar no dia-a-dia.

A multiterapia medicamentosa praticada por idosos tem sido identificada como o principal fator relacionado a segurança do paciente com relação ao aparecimento de reações adversas e interações medicamentosas. $O$ envelhecimento acarreta no aparecimento de diversas doenças como diabetes, hipertensão, osteoporose, que necessitam de tratamento contínuo, levando o idoso ao uso de vários medicamentos concomitantemente. O farmacêutico atua na atenção farmacêutica ao paciente senil, orientando 0 mesmo sobre as reações adversas e interações medicamentosas importantes, obtendo sucesso terapêutico.

\section{REFERÊNCIAS}

1 Secoli, SR et al. Polifarmácia: interações e reações adversas no uso de medicamentos por 
idosos. Revista Brasileira de Enfermagem, 2010, 63, (1): 136-140.

2 Carvalho, MFC et al. Polifarmácia entre idosos do Município de São Paulo-Estudo SABE. Revista Brasileira de Epidemiologia, 2012, 15, (4): 817-827.

3 WHO - World Health Organization. The safety of medicines in public health programmes: pharmacovigilance an essential tool. Geneva: World Health Organization; 2006.

4 Silva, GOB et al. Uso de medicamentos contínuos e fatores associados em idosos de Quixadá, Ceará. Rev. bras. epidemiol., 2012, 15, (2): 386395.

5 Silva, EA, Macedo, LC. Polifarmácia em Idosos. Saúde e Pesquisa, 2013, 6, (3): 308-315.

6 Sales, AS, Sales, MGS, Casotti, CA. Pharmacotherapeutic profile and factors associated with polypharmacy among the elderly in Aiquara, Bahia, Brazil, 2014. Epidemiologia e Serviços de Saúde, 2017, 26, (1): 121-132.

7 Bueno, CS et al. Perfil de uso de medicamentos por idosos assistidos pelo Programa de Atenção ao Idoso (PAI) da UNIJUÍ. Rev. Bras. Geriatr.Gerontol, 2012, 15, (1): 51-61.

8 Viana, AL et al. Atenção básica e dinâmica urbana nos grandes municípios paulistas, Brasil. Cadernos de Saúde Pública, 2008, 24, (1): s79-s90.

9 Lucchetti, G et al. Fatores associados à polifarmácia em idosos institucionalizados. Rev. bras. geriatr. Gerontol, 2010, 13, (1) 51-58.

10 Medeiros-Souza, $\mathrm{P}$ et al. Diagnosis and control of polypharmacy in the elderly. Revista de Saúde Pública, 2007, 41, (6):1049-1053.

11 Carvalho, ICS. Polifarmacia no Idoso in: BrunettiMonttenegro, et al Odontogeriatria: Uma visão Gerontológica. Ed. Elsevier. 2013.

12 Braveman, $\mathrm{P}$. "The social determinants of health: it's time to consider the causes of the causes." Public health reports 129.1_suppl2 (2014): 19-31.

13 Silva, AL, Ribeiro, AQ, Klein, $\mathrm{CH}$, Assis, AF Utilização de medicamentos por idosos brasileiros, de acordo com a faixa etária: um inquérito postal. Caderno de Saúde Pública, 2012, 28 (6): 10331045.

14 Barros, MBA et al. Tendências das desigualdades sociais e demográficas na prevalência de doenças crônicas no Brasil, PNAD: 2003-2008.Ciênc. saúde coletiva, 2011,16, (9): 3755-3768.

15 Machado, PEB, Matos, VC, Romeu, GA Importância da atenção farmacêutica para 0 paciente hipertenso. Infarma, 2008, 20, (3): 10-15.

16 Rocha, $\mathrm{CH}$ et al. Adesão à Prescrição Médica em Idosos de Porto Alegre, RS. Ciência e Saúde Coletiva, 2008,13, (1): 703-710.

17 Vianna, LG et al. Relação médico-paciente idoso: desafios e perspectivas. Revista Brasileira de Educação Médica, 2010, 34, (1): 150-159.

18 Mengue, SS et al. Polifarmácia e polimorbidade em idosos no Brasil: um desafio em saúde pública. Revista de saúde pública, 2016, 50, (2): 1s$13 s$.

19 Brito, GCC et al. "Relato de experiência: conscientização de idosos contra a polifarmácia e a automediacação." 11ํㅡㄹ Congresso Internacional da Rede Unida. 2014.

20 Cuentro, V et al. Prevalência e fatores associados à polifarmácia entre idosos de um hospital público. Revista Contexto \& Saúde, 2016, 16, (30): 28-35.

21 Severino, A. J. Metodologia do trabalho científico. Cortez editora, 2017, 320p.

22 Araújo, C. F. C. Uso de medicamentos inadequados e polifarmácia entre idosos do Programa Saúde da Família. Latin American Journal of Pharmacy, 2010, 29, (2): 178-184.

23 Pandolfi, M B; Paganini PL; Louzada LL. Prevalência de polifarmácia em idosos residentes em instituição de longa permanência de Brasília, Distrito Federal. Brasília méd., 2010, 47, (1): 188197.

24 Santos, T R A et al. Consumo de medicamentos por idosos, Goiânia, Brasil. Revista de Saúde Pública, 2013, 47 (1): 94-103.

25 Gomes, H O; Caldas CP. Uso inapropriado de medicamentos pelo idoso: polifarmácia e seus efeitos. Revista Hospital Universitário Pedro Ernesto, 2008, 7, (1): 125-138.

26 Lyra Jr, D P et al. Influência da propaganda na utilização de medicamentos em um grupo de idosos atendidos em uma unidade básica de saúde em Aracaju (SE, Brasil). Ciên. Saúde Coletiva, 2010, 15, (3): 3497-3505. 